\title{
Mechanical and interface properties
of biominerals: Atomistic to
coarse-grained modeling
}

\author{
Arun K. Nair, Flavia Libonati, Zhao Qin, Leon S. Dimas, and Markus J. Buehler
}

\section{Contents}

21.1 Introduction

21.2 Methods

21.2.1 Atomistic model of tropocollagen molecule and hydroxyapatite

21.2.1.1 Tropocollagen parameterization

21.2.1.2 Hydroxyapatite force field parameterization

21.2.1.3 Crystal geometry

21.2.2 Molecular dynamics simulations

21.3.1 Mechanical properties of collagen-hydroxyapatite interface

1.3.2 Effects of geometric confinement on the fracture behavior of hydroxyapatite platelets: A case study

21.3.4 Coarse grain modeling of biominerals

\subsection{INTRODUCTION}

The combination of two distinct materials, with a hard component interspersed into a soft organic matrix, is believed to be essential to many load-bearing materials, such as bone, which provide structural support for many organisms. The superior mechanical properties result from the hierarchical organization of bone, formed in a complex array of structures that spans the nanoscale to the macroscale, as described in detail in Chapter 20. Each level of organization has a crucial role in determining the overall behavior of bone at the macroscopic scale (Launey et al. 2010). From a fundamental point of view, collagen protein and hydroxyapatite (HAP) crystals are considered the primary building blocks of bone. Therefore, the mechanical behavior of these constituents as well as the interaction of the interface between them is believed to affect the mechanical response of bone at the nanoscale. However, many open questions remain with respect to the effects of various mechanisms that act on and govern the various length scales in bone.

The interface between the organic and inorganic phases in bone plays a critical role to bind the two materials together, and greatly defines the mechanical function of this composite material (Fratzl and Weinkamer 2007). The two different materials feature two extreme mechanical properties: the HAP, as a representative part of the inorganic phase, is hard and brittle, while the collagen, as a representative part of the organic phase, is soft and tends to be ductile. While the mechanical properties of any of the single material, either collagen or HAP, have been studied (Snyders et al. 2007; Gautieri et al. 2009a), the structure and interaction of these materials at their interface pose many challenging questions. Moreover, how are the thin flakes of HAP, as shown in Figure 21.1a, capable of making bone become mechanically strong and robust? To answer these questions, a deeper understanding of the interfacial functions at the molecular level is necessary. Here, we primarily discuss how the mechanical properties of the collagen-HAP interface and the molecular morphology of the crystals become critical in understanding the mechanism of the bone's mechanical function at the most fundamental level. Understanding of this mechanism is important to gain the materials' interfacial properties and go further to assess the composites' behavior with more empirical models on larger scales (Qin et al. 2012).

HAP, with the chemical formula $\mathrm{Ca}_{10}\left(\mathrm{PO}_{4}\right)_{6}(\mathrm{OH})_{2}$, has the form of a hexagonal crystal. It not only makes up to $\sim 65 \%$ wt. of the natural bone but has also found applications as an important coating material. Many modern implants, including joint replacements and dental implants, are coated with HAP (Allegrini et al. 2006). In addition to its low propensity to wear, its chemical composition may help to form a stronger bonding with bone. HAP is fully biocompatible, and its porous form is used for producing artificial bones by mimicking the mechanical properties of real bones, as well as for controlled drug-delivery systems (Sopyan et al. 2007; Kundu et al. 2010). Therefore, understanding the collagen-HAP interface is also important for designing the coating and structure of advanced materials for various bioengineering applications.

Molecular simulations provide a useful tool to investigate the atomistic-level chemomechanical properties of the 


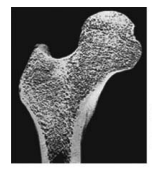

(a)
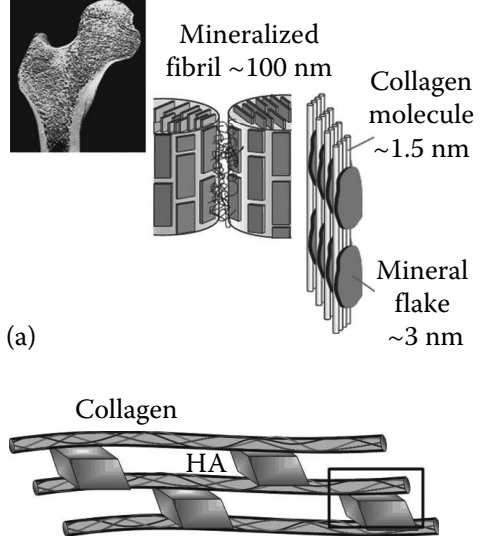

(b)

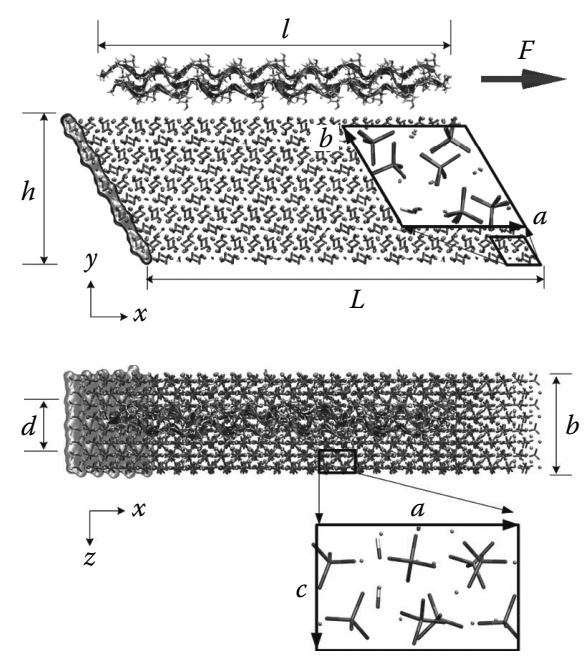

(c)

Figure 21.1 Schematic of the collagen-HAP molecular systems as reported in the study of Qin et al. (2012): (a) Microstructures of human femur bone at different scale levels (Adapted and reprinted by permission from Macmillan Publishers Ltd. Nat. Mater., Fratzl, P., Bone fracture-When the cracks begin to show, 7(8), 610-612, 2008a; Fratzl, P., Collagen: Structure and Mechanics, Springer, New York, 2008b, Copyright 2012.) (b) Schematic arrangement of the assembly of tropocollagen molecule and HAP crystals in bone. We focus on the region selected by black rectangular as shown. (c) Lateral and top views of the systems with collagen molecule lying on the Ca surface $(y+)$. A lateral total force $F$ that is parallel to the surface in $x$-direction is applied to the three alpha carbon atoms at the right-end of the tropocollagen segment, while a single layer of atoms at the left-end of the HAP is fixed as shown by the bordering outlines. The OH surface is the bottom surface ( $y-$ ) of the HAP crystal in this panel. We zoom in to show the projection of a unit cell of the HAP with its three lattice constants $a, b$, and $c$. (Panels $b$ and $c$ : Reprinted from Qin, Z. et al., Langmuir, 28(4), 1982, 2012.)

organic-inorganic interfaces and its correlation with the overall mechanical behavior of the composite materials. Studies to this level with detailed mechanisms are still difficult or impossible to achieve with experimental techniques alone. Atomistic simulations have also been recently used to gain insights into the mechanical properties of HAP (Snyders et al. 2007) and its interfacial property with short peptides. Previous works have used atomistic simulations to investigate the load-deformation behavior of tropocollagen molecules with HAP in proximity of their terminals (Bhowmik et al. 2009), the adsorption energy of tropocollagen molecules such as the common Gly-ProHyp segment (Almora-Barrios and deLeeuw 2010b), and the description of model systems enamel (Zahn and Duchstein 2011). Those models are still quite far from the basic unit of the natural bone configuration.

In this chapter, we present models of bone-like composites, applied to shed light on the mechanisms by which HAP can provide stiffness to the collagen matrix. We first discuss the methodology that is utilized to describe the interactions between collagen molecules, HAP crystals, and the interactions between collagen and HAP. We then discuss the mechanical properties of a collagen-HAP composite, followed by a study of size effects in HAP crystals in resistance to fracture. Our basic model is set up by considering a unit cell of the nanostructures of tropocollagen and HAP crystal, as shown in Figure 21.1b, and their interface is parallel to the tropocollagen axis. By applying mechanical force to this model and studying its response, the model can be used to understand the tropocollagen-HAP interface properties under extreme mechanical conditions. We also introduce a microfibril model of bone and review the results for tensile tests. The latter study corresponds to nanoscale deformation mechanisms. Finally, a coarse grain model for bone is presented, which aims at uncovering macroscale level deformation mechanisms, and also leads a way to translate the structure of bone into synthetic materials that mimic some of the key mechanical traits.

\subsection{METHODS}

\subsubsection{ATOMISTIC MODEL OF TROPOCOLLAGEN MOLECULE AND HYDROXYAPATITE}

In atomistic modeling, the interactions between tropocollagen molecules and HAP at interfaces are decomposed into basic energy contributions, including van der Waals interactions, Coulombic interactions, and hydrogen bonds. The van der Waals interaction is computed pairwise for all the atom pairs in the tropocollagen molecule and HAP, while the Coulombic interactions mainly exist between charged side chains (e.g., $\mathrm{NH}^{3+}$ and $\mathrm{COO}^{-}$) and surface ions of $\mathrm{HAP}$ (e.g., $\mathrm{Ca}^{2+}, \mathrm{PO}_{4}^{3-}$, and $\left.\mathrm{OH}^{-}\right)$. Hydrogen bonds are formed between the uncharged polar side chains (e.g., $\mathrm{OH}$ in $\mathrm{Hyp}$ ) and $\mathrm{OH}$ groups in HAP.

We build the atomistic structure of the tropocollagen molecule by using the software THeBuScr (triple helical collagen building script) (Rainey and Goh 2004). We focus on the simplest model of collagen, with only Gly-Pro-Hyp triplets on each of the three chains. The tropocollagen molecule model that we use, [(GlyPro-Hyp) $\left.)_{10}\right]_{3}$, is composed of only 30 amino acids per chain in order to reduce computational costs; such a length is comparable to the length used in previous studies (Buehler 2006; Gautieri et al. 2009a,b). Because the tropocollagen length $(9 \mathrm{~nm})$ is shorter than its persistence length $(10-20 \mathrm{~nm})$, entropic effects are not significant for our model. The computational model is developed with the aim of elucidating the generic behavior and deformation of a system containing the interaction between a single tropocollagen and a HAP nanocrystal, and as such is designed to be simple and not intended to be a direct representation of the actual bone nanostructure with a specific sequence. Our model 
enables us to perform a systematic study of bone nanomechanics from a fundamental point of view.

\subsubsection{Tropocollagen parameterization}

Collagen is the sole protein that features hydroxyproline (HYP), a nonstandard amino acid, resulting from hydroxylation of proline. Since it is rarely found, HYP is not parameterized in common biomolecular force fields like CHARMM (Brooks et al. 1983). A force field set for HYP has been developed by using quantummechanical simulations and subsequently deriving the atomistic parameters that best match the quantum-mechanics calculation (Park et al. 2005), with a particular focus on the correct modeling of the pucker of the HYP ring.

\subsubsection{Hydroxyapatite force field parameterization}

Most force fields for biomolecular simulations, like CHARMM (Brooks et al. 1983), do not include parameters for crystalline minerals such as HAP. Therefore, in order to model biomolecular systems including HAP, we extended the CHARMM force field. We use bond, angle, and dihedral parameters as reported earlier (Hauptmann et al. 2003), which are based on both quantummechanics calculations and empirical data. Therefore, for nonbonded terms, we use data from the study of Bhowmik et al. (2007a) in which the authors fitted the Born-Mayer-Huggins model (Hauptmann et al. 2003) with a simpler Lennard-Jones potential in CHARMM.

\subsubsection{Crystal geometry}

We generated the hexagonal HAP crystal unit cell by using Materials Studio 4.4 (Accelrys, Inc.), with the following lattice parameters: $a=9.4214 \AA, b=9.4214 \AA, c=6.8814 \AA, \alpha=90^{\circ}$, $\beta=90^{\circ}$, and $\gamma=120^{\circ}$. Based on this unit cell ( 44 atoms per unit cell), HAP crystals of varying thicknesses of $h=0.7,1.4,3.5$, and $4.2 \mathrm{~nm}$ are generated for the purpose of this study. In our study, we focus on the interaction on the (010) plane, because this plane is dominant in the morphology of the biological material, due to the growth direction of the collagen matrix (Almora-Barrios and deLeeuw 2010a). Another reason to study this plane is that the surface charge for the two opposite surfaces of this plane is not neutral but negatively charged on the $\mathrm{OH}$ surface and positively charged on the Ca surface. Since the electrostatic interaction between the tropocollagen molecule and mineral part plays an important role to stabilize the interface, those two surfaces provide two extreme cases for our study. Moreover, since the (100) plane has similar geometric and chemical properties as the (010) plane and the surface charge of the (001) plane is neutral, by studying the two surfaces of the (010) plane, we may gain more representative results over the other two surfaces.

\subsubsection{MOLECULAR DYNAMICS SIMULATIONS}

Molecular dynamics calculations are performed using the LAMMPS code (Plimpton 1995) and the modified CHARMM force field. Lennard-Jones and Coulomb interactions are computed with a switching function that truncates the energy and force smoothly to 0 from 8 to $10 \AA$. This cutoff range is selected to include at least one complete lattice in the thickness direction. We have tested cutoff lengths longer than this value, and the tensile modulus and strength of the collagen-HAP model are not affected by this increment. The constructed collagen-HAP model is first geometrically optimized through energy minimization; then, an $N V T$ (a canonical ensemble where moles, $N$, volume, $V$, and temperature, $T$, are conserved) equilibration is performed for 200 ps. The system temperature is set to constant $300 \mathrm{~K}$ with Langevin thermostat. After equilibration, we observe that the root-mean-square deviation of the crystal is stable and that no major changes occur in the crystal structure, confirming the reliability of the extended CHARMM force field. Visual molecular dynamics (VMD) (Humphrey et al. 1996) is a convenient tool for visualization, used here to show the snapshots of simulation and to compute hydrogen bonds. We count the hydrogen bonds within a cutoff distance of $3.5 \AA$ and an angle range of $30^{\circ}$. We use MATLAB ${ }^{\circledR}$ (Mathworks, Inc.) to analyze the results of the mechanical testing.

\subsection{CASE STUDIES USING MECHANICAL SIMULATIONS}

\subsubsection{MECHANICAL PROPERTIES OF COLLAGEN- HYDROXYAPATITE INTERFACE}

Using the models of tropocollagen molecule and HAP as shown in Figure $21.1 \mathrm{~b}$ and c, with the geometric parameters as summarized in Table 21.1, we study the effects of mineral surface, mineral thickness, and hydration state systematically, as reported by Qin et al. (2012). To obtain the force displacement relations, we use steered molecular dynamics (SMD) for loading the tropocollagen molecule and by fixing the left-end part of the HAP crystal and pulling the center of mass of the right-end alpha-carbon atoms of the tropocollagen molecule. The pulling velocity is set to $0.01 \AA / p s$ (equivalent to $1 \mathrm{~m} / \mathrm{s}$ ), similar to that used in earlier simulation studies of the protein's tensile properties (Gautieri et al. 2009c; Qin et al. 2009). We keep a record of the applying force $F$ as a function of the displacement of the SMD loading point $d x$. In the postprocessing stage, we calculate the applied stress to the end of the tropocollagen molecule via $\sigma=F / A$, where $A=\pi d^{2} / 4$ is the cross-section area of the tropocollagen molecule, where $d$ is the average diameter as shown in Figure 21.1c. Here, $A$ only counts

Table 21.1 Basic geometric parameters of tropocollagen molecule and HAP for modeling, as shown in Figure 21.1

\begin{tabular}{|l|l|c|l|}
\hline MATERIAL & CRYSTAL TYPE & $\begin{array}{c}\text { GEOMETRIC } \\
\text { PARAMETERS }(\mathrm{nm})\end{array}$ & BASIC UNIT \\
\hline Tropocollagen molecule & Protein triple helix & $\begin{array}{l}d=1.5 \\
l=9.3\end{array}$ & GPO \\
& & $\begin{array}{l}b=0.7-4.2 \\
b=2.5\end{array}$ & $\mathrm{Ca}_{10}\left(\mathrm{PO}_{4}\right)_{6}(\mathrm{OH})_{2}$ \\
& Hexagonal crystal & & \\
& & $L=10.5$ & \\
\hline
\end{tabular}



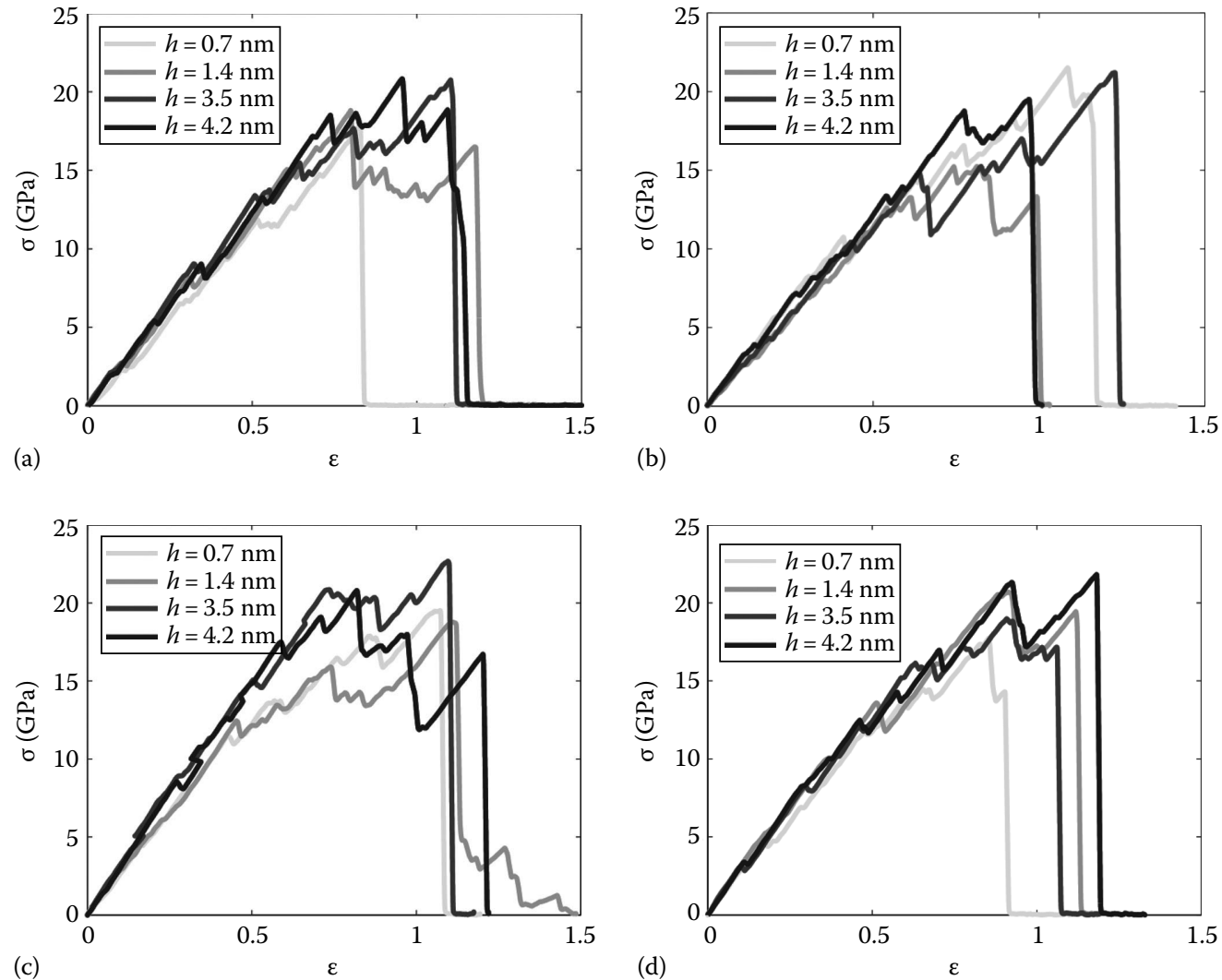

Figure 21.2 Stress-strain curves for collagen-HAP composites for HAP thicknesses $h$ as indicated in the legends (Qin et al. 2012): (a) Stressstrain curves for $\mathrm{Ca}$ surface and (b) for the $\mathrm{OH}$ surface in dry conditions. (c) Stress-strain curves for Ca surface and (d) for the OH surface in hydrated conditions. (Reprinted from Qin, Z. et al., Langmuir, 28(4), 1982-1992, 2012.)

the cross-section of the tropocollagen molecule, because the force in our model is directly applied to the tropocollagen molecule end for the simulation set up. The tensile deformation is measured by the engineering strain calculated via $\varepsilon=d x / l$, where $l$ is the length of the tropocollagen molecule.

First, we address the effect of HAP thickness $h$ on the mechanical response of the model under tensile force. The forceextension curves in our simulations corresponding to various thicknesses and chemical surfaces of the HAP are summarized in Figure 21.2. All curves display a linear region from the beginning, followed by a "bumpy" region before failure of the material occurs. We observe that the deformation of the model in the first region originates from the extension of the atomic interactions, and rarely from the sliding between the tropocollagen molecule and HAP. For the second "bumpy" region, we observe that the collagen chains, after fully stretching, start to slide on top of the HAP surface. Notably, this sliding mechanism is consistent with the experimental observations (Gupta et al. 2013), where the intrafibrillar sliding between mineral and collagen leads to plastic strain in the fibril and the tissue level. Such a sliding mechanism occurs in a discontinuous way because of the atomically rough surface of the electrostatic interactions and hydrogen-bond forming patterns. The hydrogen bond analysis by Qin et al. (2012) shows that a difference in the number of hydrogen bonds for dry conditions and presence of water implies that the number of hydrogen bonds between the tropocollagen molecule and HAP contributes to the interaction force. We quantitatively analyze those strain-stress curves by focusing on the modulus and strength of this interface.
An analysis of tensile modulus $E$ of the system for different surfaces and solvent conditions shows that the existence of HAP significantly increases with the stiffness of the system by comparing the tensile modulus to that of the pure collagen triple helix. We fit those results mathematically by using the exponential function

$$
E=E_{\infty}-\left(E_{\infty}-E_{C O L}\right) \exp \left(\frac{-h}{h_{E 0}}\right)
$$

where

$E_{\infty}$ is the converged value of the tensile modulus for larger $h$

$E_{C O L}(4.64 \mathrm{GPa}$, which agrees with the result of an earlier simulation study (Gautieri et al. 2009a)) is the tensile modulus of pure tropocollagen molecule

$h_{0}$ is a length constant as the characteristic thickness

It shows that for HAP crystals with a thickness beyond $h_{0}$, increasing the thickness can only increase the stiffness of the system up to $50 \%$. We summarize $E_{\infty}$ and $h_{0}$ for each of the two surfaces with and without water around the interface in Table 21.2. It can be seen that $E_{\infty}$ is rather insensitive on the surface or hydration state, reaching a value of $\approx 31 \mathrm{GPa}$. On the other hand, the tensile modulus is shown to be dependent on the crystal thickness, in particular, for the Ca-rich surface in dry condition, where the tensile modulus in the case of the thinnest crystal $(0.7 \mathrm{~nm})$ is found to be about half the convergence value. Also, in the case of $\mathrm{OH}$-rich surface (dry conditions), the thickness has little or no influence, possibly due to the higher 
Table 21.2 Values of the parameters of the tensile modulus and strength of collagen-HAP interfaces as functions of the thickness of the HAP, defined in Equations 21.1 and 21.2

\begin{tabular}{|c|l|c|c|c|c|}
\hline \multirow{2}{*}{\multicolumn{2}{|c|}{ PARAMETER }} & \multicolumn{2}{c|}{ TENSILE MODULUS } & \multicolumn{2}{c|}{ TENSILE STRENGTH } \\
\cline { 2 - 6 } & $E_{\infty}(\mathrm{Gpa})$ & $h_{E 0}(\mathrm{~nm})$ & $\sigma_{\infty}(\mathrm{Gpa})$ & $h_{\sigma 0}(\mathrm{~nm})$ \\
\hline \multirow{2}{*}{ Ca surface } & No water & 30.16 & 0.86 & 20.79 & 0.84 \\
\cline { 2 - 6 } & With water & 31.72 & 0.51 & 21.41 & 0.68 \\
\hline \multirow{2}{*}{ OH surface } & No water & 31.72 & 0.02 & 19.37 & 0.02 \\
\cline { 2 - 6 } & With water & 31.87 & 0.36 & 20.62 & 0.60 \\
\hline
\end{tabular}

number of collagen-HAP hydrogen bonds formed. In wet cases, the difference in the thickness effect (suggested by the differences by $E_{\infty}$ and $h_{0}$ ) is less strong, due to the presence of water-mediated hydrogen bonds at the interface. This statement is supported by the evidence that water has a very strong attractive interaction with both collagen and HAP, especially for HAP (Katti et al. 2010), while at the same time, the existence of water can decrease the direct mechanical connection between the tropocollagen molecule and HAP with a lubricant effect (Gupta et al. 2006). Thus, by combining the analysis to our simulation results of $E_{\infty}$ for each of the two surfaces, we can conclude that the existence of water increases the collagen-HAP interaction at the Ca-rich surface (indicated by the decreased $h_{0}$ ), but decreases the collagen-HAP interaction at the $\mathrm{OH}$-rich surface (indicated by the increased $h_{0}$ ), leading to a more uniform $E-h$ relationship for different surfaces as summarized in Table 21.2.

We fit the maximum stress, $\sigma_{C}$, reached by the strain-stress curve, as a function of the thickness $h$ by using

$$
\sigma_{C}=\sigma_{\infty}-\left(\sigma_{\infty}-\sigma_{C O L}\right) \exp \left(\frac{-h}{h_{\sigma 0}}\right)
$$

where

$\sigma_{\infty}$ is the converged maximum stress (Qin et al. 2012)

$\sigma_{C O L}(13 \mathrm{GPa}$ from an earlier simulation study (Buehler 2006)) is the maximum stress of pure tropocollagen molecule

$h_{\sigma}$ is the characteristic thickness

It shows that for HAP crystals with a thickness beyond $h_{\sigma}$, increasing the thickness can only increase the strength of the system up to $15 \%$. We summarize $\sigma_{\infty}$ and $h_{\sigma}$ for each of the two surfaces with and without water around the interface in Table 21.2. It can be seen that $\sigma_{\infty}$ is rather insensitive on the surface or hydration state, reaching a value of $\approx 20 \mathrm{GPa}$. Similarly, we find a significant dependence of $\sigma_{C}$ on $h$ (shown for large $h_{\sigma}$ ) for the Ca-rich surface in dry conditions. Also, in the case of $\mathrm{OH}$-rich surface (dry conditions), the thickness has little or no influence. In the wet cases, the difference in the thickness effect (indicated by the differences in $\sigma_{\infty}$ and $h_{\sigma}$ ) is less strong for these two different surfaces. This result agrees to the conclusion that the existence of water mediates the collagen-HAP interface and leads to a more uniform $\sigma_{C}-h$ relationship for different surfaces. The result also makes it clear that since the tensile strength of the pure tropocollagen molecule is weaker than this theoretical prediction of the collagen-HAP composite, the rupture event happens by breaking the tropocollagen molecule instead of by breaking the interface. The reason that our breaking force is larger than the tropocollagen strength is that the CHARMM force field we used here does not account for covalent bond breaking. Therefore, to make a more precise description of the rupture process of the interface (the competing process between collagen rupture and sliding apart at the interface), one may need a more fundamental reactive force field for the atomic interactions (Buehler 2006).

To summarize, this section discussed the effect of mineral surface, mineral thickness, and hydration state on the mechanical properties of collagen-mineral interface. We find that thin flakes of HAP crystals less than $1 \mathrm{~nm}$ can significantly improve the tensile modulus of the pure tropocollagen molecule. This observation is likely due to the fact that the tensile modulus of the HAP is much higher than that of the tropocollagen molecule, making the composite much stiffer than pure collagen. This result agrees with the experimental observations in which mineralized tendon shows a much higher tensile modulus (it can reach a factor of 10 times) than the unmineralized tendon (Weiner and Wagner 1998). On the other hand, the tensile modulus of the biomineral interface does not depend on the surface and hydration state, reaching a value of $\approx 31 \mathrm{GPa}$ for increasing thicknesses. Specifically, the tensile modulus converges at a thickness of less than $2 \mathrm{~nm}$, which agrees with the experimental observations that mineralized collagen structures in most forms of the natural morphology of bones are composed of collagen and thin HAP flakes of uniform thickness that varies between 1 and $4 \mathrm{~nm}$ for different bone types (Weiner and Wagner 1998; Fratzl 2008a; Hu et al. 2010). Such an agreement between simulations and experimental observations shows that the nanoscale structure of the collagen-HAP interface is important in defining the mechanical properties of bone. Our simulation results also show that the existence of water mediates the tensile modulus and strength of the collagen-HAP interface, leading to more homogeneous tensile modulus and tensile strength for various HAP surfaces with different chemical compositions. These results imply that the microscopic structure of bone evolved in nature largely according to its mechanical requirement to achieve its crucial function in supporting and protecting the body.

\subsubsection{EFFECTS OF GEOMETRIC CONFINEMENT ON THE FRACTURE BEHAVIOR OF HYDROXYAPATITE PLATELETS: A CASE STUDY}

The behavior of many biological materials is strongly affected by the geometric confinement at the nanometer length scale. As a result, the mechanical properties such as the strength and fracture toughness are strongly dependent on the characteristic size. 
For instance, the human bone is well-known for its resistance to fracture, and it has been suggested that this mechanical property has its origin in a multitude of deformation and toughening mechanisms that occur at different scales, from the nanoscale to the macroscopic one (Ritchie et al. 2004, 2009; Dubey and Tomar 2008; Launey et al. 2010; Sen and Buehler 2011). The load transfer between the mineral and the protein phase has been described by the tension-shear model (Gao et al. 2003), where the mineral platelets carry most of the tensile load, and the protein matrix transfers the load between the platelets by shear (Ji and Gao 2004a,b; Gupta et al. 2005; Buehler et al. 2006; Gao 2006).

As was confirmed using a series of molecular dynamics simulations of HAP crystals in the study of Libonati et al. (2013), the geometric characteristics of the crystals have a strong influence on the mechanical properties. The elongated platelet shape leads to a high surface to volume ratio, ensuring a greater interaction with the collagen protein and enhanced mechanical properties. Indeed, using molecular models, we can directly address the effect of a systematic variation of the characteristic HAP crystal size on its mechanical behavior in the presence of an edge crack (Libonati et al. 2013). The effect of confining the size at the nanoscale on fracture response is studied by performing a series of in silico mechanical tests of tiny HAP samples of different sizes, with an edge crack. The dimensions of the samples $(30.1 \times 2.1 \times 2 h \mathrm{~nm})$ are chosen so as to be similar to the HAP crystal platelets found in the bone. These platelets are generally tens of nanometers in width and length and 2-3 nm in thickness (Rho et al. 1998).

As presented in the study of Libonati et al. (2013), we consider a thin slab of HAP with an edge crack, which extends for half of the slab width, and we systematically vary the characteristic crystal size (i.e., the height of the crystal, 2 h), keeping the sample length, the crack length, and the loading and boundary conditions constant for all the studied cases. An example of the tested sample is given in Figure 21.3a. The samples are created by replicating the unit cell (see Section 21.2.1 for the simulation cell dimensions) 32 times in the $x$-direction, 3 times in the $z$-direction, and $n$ times in the $y$-direction, where $n$ varies from 4 to 14 . The HAP samples are generated by systematically

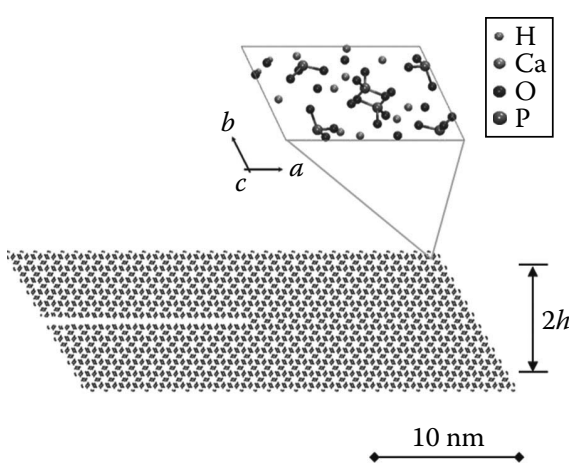

(a)

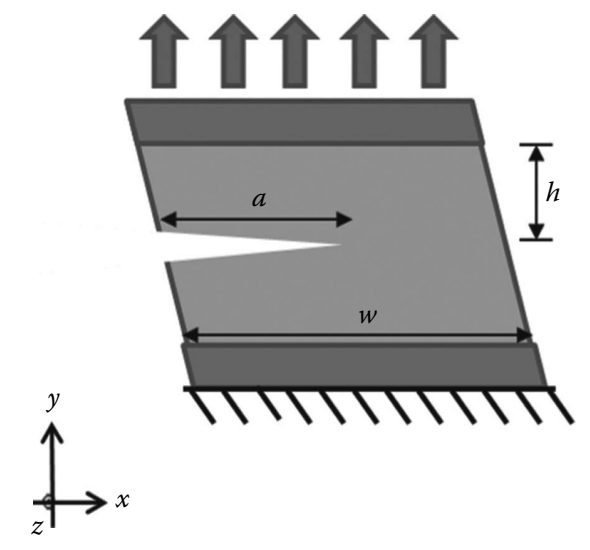

Figure 21.3 Geometry, dimensions, and loading and boundary conditions of a cracked HAP slab: (a) Snapshot of the sample in its initial geometry. The inset shows a zoomed view of the HAP unit cell, in a view parallel to the (001) plane (each atom is represented with a different size). (b) Model used for the atomistic study of fracture of HAP. Different samples are created by varying the sample aspect ratio ( $h / w)$. The width $(w)$ and the crack length (a) are fixed for all specimens, and the crack extends over half of the platelet width. (Reprinted from Libonati, F. et al., J. Mech. Behav. Biomed. Mater., 20, 184-191, 2013.)

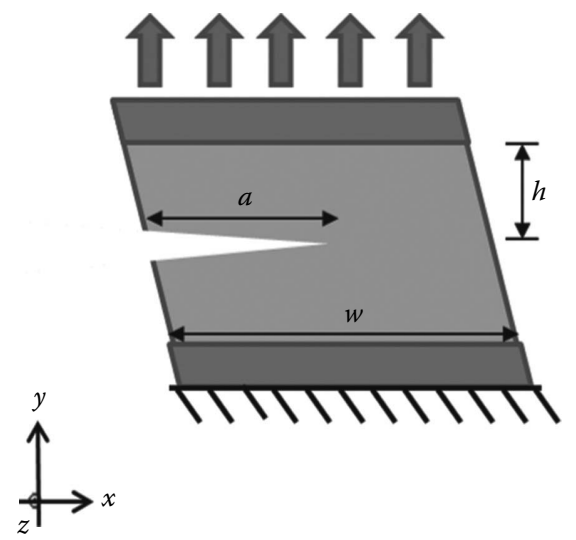

increasing the height by two unit cells in the $y$-direction. The crack extends in the basal plane (001) with the main axes on the (010) plane. In all the cases, we consider a hexagonal closepacked (HCP) crystal, with $x=[100], y=[010]$, and $z=[001]$. For simulations, we use an extended CHARMM force field discussed in Section 21.2.1.

By using a simple mechanical scheme, as shown in Figure 21.3b, each sample is clamped at the bottom and pulled at the top, in displacement control mode, applying a velocity of $1 \AA$ /fs. We adopt a quasi-static loading approach, consisting of several loading steps followed by relaxation, through an energy minimization scheme, until failure occurred. The virial stress, calculated from the atomic positions and forces, and the engineering strain are used to obtain the stress-strain response. The mechanical properties (i.e., stiffness, maximum strength, strain at failure, etc.) are calculated from the stress-strain data. Stress maps are created by plotting the virial stresses (Tsai 1979; Zimmerman et al. 2004) on each atom, by means of the AtomEye tool (Li 2003). For analysis, the atomistic virial stress data computed in a thin strip ahead of the crack tip are averaged, and then fitted by using a power law of the form

$$
y=(1-t) e^{-\beta_{i} x}+t,
$$

where $t$ represents the asymptotic value for each sample; $\beta_{i}$ is defined in two ways:

$$
\beta_{1}=\frac{y_{2}-y_{1}}{x_{2}-x_{1}} \cdot(t-1)^{-1}
$$

and

$$
\beta_{2}=\frac{1}{x_{2}} \cdot \ln \left(\frac{1-t}{y_{2}-t}\right) .
$$

$x_{1}$ and $x_{2}$ represent the $x$-coordinates of the first two data, in each sample, and $y_{1}$ and $y_{2}$ represent the stress values of the first two data, in each sample.

(b) 


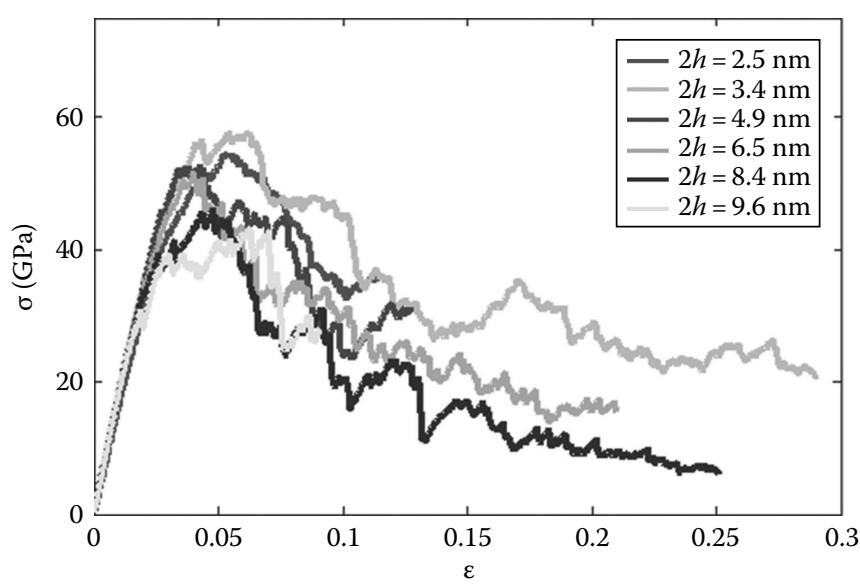

Figure 21.4 Stress-strain plot of the tested samples (Libonati et al. 2013). The stress plotted is $\sigma_{y y}$ and the strain represents the applied tensile strain in the $y$-direction. The initial slope of the stress-strain curve is not affected by the size of the sample, while the strength decreases as the sample height grows. (Reprinted from Libonati, N. et al., J. Mech. Behav. Biomed. Mater., 20, 184-191, 2013.)

The results revealed that a decrease in the crystal size causes a change in the mechanical behavior of this material and the occurrence of new phenomena that are not present at larger scales: 1. A general increase in the mechanical properties (i.e., strength, toughness modulus)

2. A change in the failure mode, from a crack-driven failure in larger samples to a more spread failure mode in the smaller ones

3. A change in the stress field, from a heterogeneous stress field, characterized by a high stressed area near the crack tip, to a more homogeneous stress field

By varying the characteristic crystal size $2 h$, we observe a variation in the mechanical response and properties (i.e., strength and toughness modulus), as shown in Figure 21.4, where the stress-strain curves of the tested samples are depicted. In all the cases, it is possible to recognize an initial linear region, where the

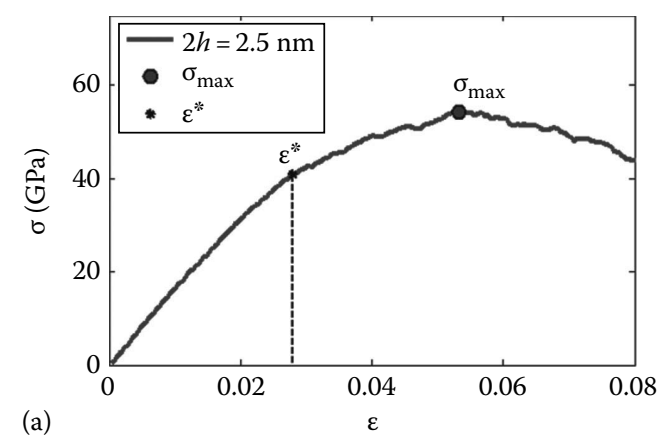

(a)

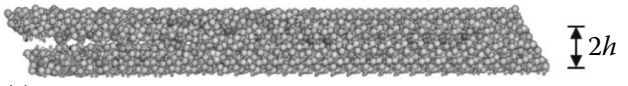

(c) material is slightly deformed in the elastic field. In this region, the material behavior is not dependent on the sample size; for instance, we observe that the slope of the stress-strain curves is similar for all cases; so, the stiffness of the sample is not affected by the sample size. This linear region is followed by a second region, characterized by a deviation from linearity, where the stress slowly increases until a maximum has been reached, and a subcritical propagation of the defect occurs. Indeed, initially we do not observe a clear crack propagation mechanism. Then, as the load increases, different phenomena besides crack opening occur. For small-sized cases, many defects emerge in different parts of the samples, growing until the maximum stress is reached. In this case, there is no sudden drop of the stress, like in larger samples, but it is "stepwise" decreasing. For larger samples, we find that failure is more localized around the crack tip region.

Beyond the maximum stress, failure occurs in different modes depending on the sample size. For small samples, it occurs more gradually, and it is not characterized by a clear crack path. Damage is widespread due to the formation and interaction of many small defects over the whole volume. The growth of these defects and their interaction lead to the global failure of the slab. By increasing the sample size, we observe a clearly distinguished crack path (Libonati et al. 2013). The difference in the failure mode is probably due to the different stress distributions reached in the various samples. Another effect of confining the size at the nanoscale is a change in the stress distribution. We observe that by decreasing the sample height, the stress concentration that characterizes the larger samples disappears. The more homogeneous stress distribution explains why small samples are characterized by a more spread failure damage and a slower failure mode. Figure 21.5 shows the stress-strain curves and stress fields for two samples of different sizes, confirming that there is a clear difference between the two cases. In the smaller sample (Figure 21.5c) with $2 h=2.5 \mathrm{~nm}$, the stresses are homogenously distributed, whereas in the larger one (Figure 21.5d) with $2 h=8.4 \mathrm{~nm}$, a concentration of higher stresses is clearly visible
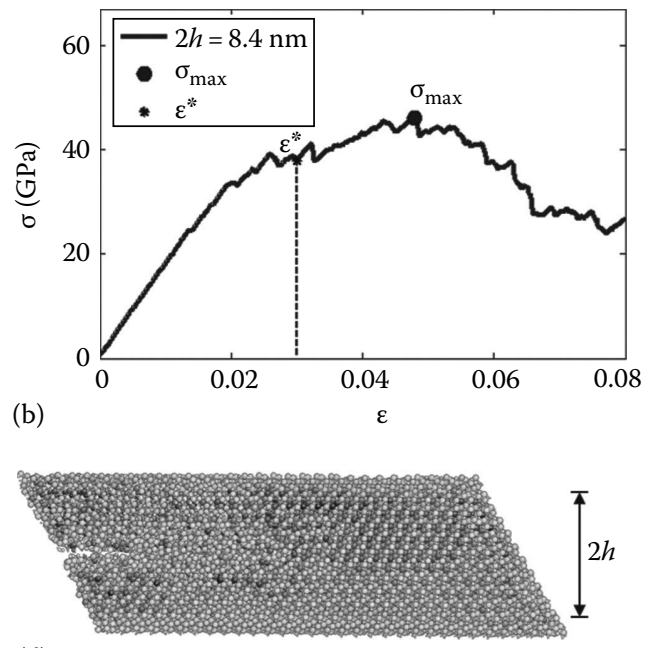

(d)

Figure 21.5 Stress-strain curve of two samples: (a) $2 h=2.5 \mathrm{~nm}$ and (b) $2 h=8.4 \mathrm{~nm}$. The lower panels show maps of the stress distribution for two cases, (c) $2 h=2.5 \mathrm{~nm}$ and (d) $2 h=8.4 \mathrm{~nm}$. The maps show the stress field (in the middle section of each sample) at the critical strain $\varepsilon^{\star}$, which is highlighted in the earlier curves. The maximum stress $\left(\sigma_{\max }\right)$, reached before failure occurs, is highlighted in the two curves.

(c) Depiction of a homogeneous stress distribution. (d) High stress concentration that forms ahead of the crack tip. (Reprinted from Libonati, N. et al., J. Mech. Behav. Biomed. Mater., 20, 184-191, 2013.) 


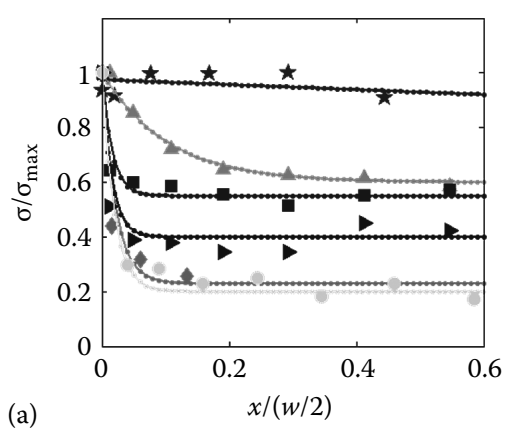

(a)

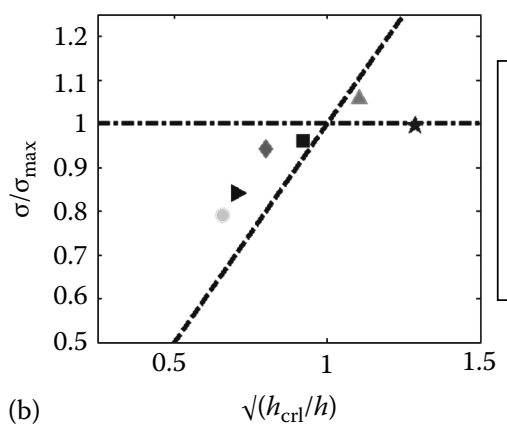

$\star 2 h=2.5 \mathrm{~nm}$

A $2 h=3.4 \mathrm{~nm}$

- $2 h=4.9 \mathrm{~nm}$

- $2 h=6.5 \mathrm{~nm}$

- $2 h=8.4 \mathrm{~nm}$

$2 h=9.6 \mathrm{~nm}$

--- Griffith theory

-.- Flaw tolerance

Figure 21.6 Stress fields for varying crystal dimensions (Libonati et al. 2013): (a) Stress field reached from the crack tip to the far field for different sample sizes. The longitudinal stress field, averaged over a thin strip in the middle and over the entire thickness, is plotted against the $x$-direction (the zero $x$-coordinate represents the crack tip). The values on both the axes are normalized by the maximum values, respectively. The continuous lines represent power-law fits to the atomistic data. (b) Normalized maximum strength over the normalized sample size. Comparisons with the Griffith theory prediction are given, showing a clear deviation at small length scales below $h_{c r l}=4.15 \mathrm{~nm}$. (Reprinted from Libonati, N. et al., J. Mech. Behav. Biomed. Mater., 20, 184-191, 2013.)

ahead of the crack tip. This phenomenon is directly seen in Figure 21.6, where we plot the longitudinal stress distribution in a thin strip ahead of the crack tip just before failure occurs. The graph shown in Figure 21.6a confirms that the small sample is characterized by a quasi-homogeneous stress distribution, while the larger ones are characterized by a high-stressed region near the crack tip. The larger the size, the more localized is the area of high stress concentration. This analysis reveals that the stress field becomes increasingly homogeneous as the size is reduced.

From the stress-strain curves depicted in Figure 21.4, we calculate the strength, defined as the maximum stress reached during the test; we find that it is clearly affected by the size, being generally higher for smaller samples. As expected, the mechanical properties (i.e., the strength) also show a trend until a certain dimension is reached by the crystal. Below this characteristic size, the strength seems not to be affected by the variation of the size, hence approaching a constant value. Indeed, on the basis of the results presented earlier (e.g., failure mode, stress field, stress distribution from the crack tip to the far field), we observe that a distinct change in the mechanical behavior occurs at around $2 h=4.15 \mathrm{~nm}$. By considering the determined values of the strength (normalized by the maximum one) as a function of the inverse of the square root of the sample size, $\sqrt{h_{c r} / h}$, a bilinear trend is found (Figure 21.6b). Moreover, by comparing the data with the prediction of the Griffith's theory, we find that this theory is valid only for samples larger than the critical size, whereas for the smaller ones this theory does not hold, in agreement with the hypothesis put forth in the study by Gao et al. (2003). These findings corroborate the concept that, at the nanoscale, flaw tolerance occurs in HAP crystals below a critical size on the order of a few nanometers.

This study (Libonati et al. 2013) provided several important insights into the phenomena that occur at the nanoscale and on the different mechanisms governing the fracture at the nanoscale, where the concepts of stress concentration, crack-driven and brittle failure mode, and size-affected mechanical properties are replaced by quasi-homogeneous stress distribution, larger strain to failure, flaw tolerance, and constant mechanical properties, regardless of the presence of defect. This study may have large impact in understanding the high toughness of bone and bone-like materials, although they are mainly made of a brittle material.

\subsubsection{ATOMISTIC MODELING OF A MINERALIZED COLLAGEN MICROFIBRIL}

The model that was presented in Section 21.3.1 is limited to a single tropocollagen molecule ( $\sim \mathrm{nm}$ scale). However, the assembly of several collagen molecules that form collagen fibrils has a diameter on the order of $100 \mathrm{~nm}$ (Ritchie et al. 2009). The knowledge about how collagen fibrils and HAP crystals interact at the molecular scale, and how they deform as an integrated system under external stress, are not well-understood. Several attempts have been made to develop a molecular model of bone resulting in a recently reported full-atomistic model of a mineralized collagen fibril that will be discussed here (Nair et al. 2013). The earlier studies fell short to providing a complete three-dimensional, chemically and structurally accurate model of the interactions of collagen with the mineral phase (Boskey 2003; Bhowmik et al. 2007b, 2009; Zhao et al. 2012). Coarse grain modeling (Buehler 2007) of nascent bone showed that the mineral crystals provide additional strength and also increases the Young's modulus and fracture strength. While these models provided some insight into the mechanics of bone, they failed to capture the atomic-scale mechanisms and did not allow for a direct comparison with the experimental work (e.g., in situ x-ray analysis of bone deformation [Weiner and Wagner 1998; Jager and Fratzl 2000; Fratzl et al. 2004; Gupta et al. 2006; Fratzl and Weinkamer 2007; Fratzl 2008b]).

The nucleation and growth of minerals in collagen fibril is a complex phenomenon (Colfen 2010) and involves timescales on the order of microseconds, and hence cannot be simulated directly by molecular dynamics with the current computational capabilities. Hence, in this model we apply an in silico mineralization scheme (Nair et al. 2013) that allows us to fill an initially unmineralized collagen fibril structure, up to a maximum mineral density of $40 \%$. The geometry and composition of the model are illustrated in Figure 21.7, indicating varied levels of mineral content. The $0 \%$ case corresponds to the nonmineralized collagen microfibril and also shows the gap and the overlap regions (Figure 21.7), which emerge from the geometrical arrangement of collagen protein molecules. The $D$-period corresponds to the periodicity typically observed in collagen fibrils. The $20 \%$ and $40 \%$ 


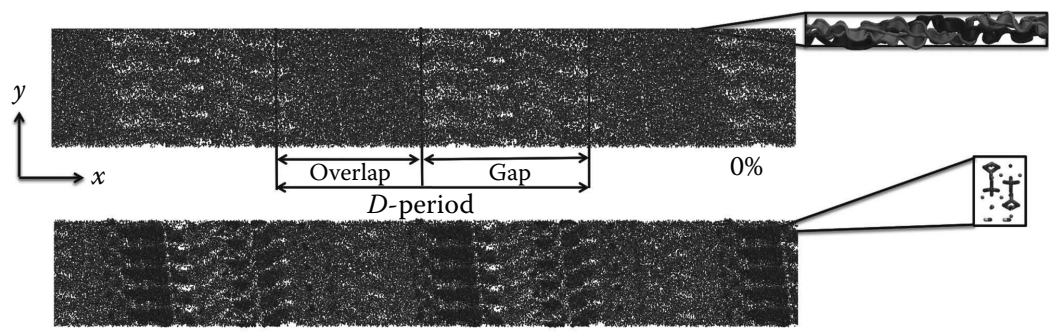

$20 \%$

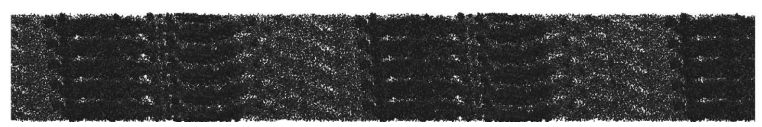

$40 \%$

Figure 21.7 Full atomistic model of mineralized collagen fibrils as reported in the study of Nair et al. (2013). Collagen microfibril model with $0 \%$ mineralization (inset shows collagen triple helix structure), $20 \%$ mineral content (inset shows a HAP unit cell), and $40 \%$ mineral content. For mineralized cases, the dark region shows the presence of minerals in the gap and overlap regions. Inset shows that the HAP crystals are arranged such that the $c$-axis of the crystal aligns with the fibril axis. The HAP crystal comprises of $\mathrm{C}_{\alpha}$ atoms, $\mathrm{OH}$ group and tetrahedron structure shows the $\mathrm{PO}_{4}$ group. (Reprinted from Nair, A.K. et al., Nat. Commun., 4, 1724, 2013.)

mineral density cases correspond to the two different mineral concentrations in the collagen microfibril.

As shown in Figure 21.8a, the mineral is deposited predominantly in the gap region along the fibril axis, with sparse deposition in the overlap region. Figure $21.8 \mathrm{~b}$ shows a detailed analysis of the distribution of mineral crystals for $20 \%$ and $40 \%$ mineral densities. We perform a direct comparison with the experimental results that reported the distribution of mineral density as a function of the fibril axis (Nudelman et al. 2010), and plot the mineral distribution of $40 \%$ case normalized to its maximum value along with the experimental results along the fibril axis (Figure 21.8c). In good agreement with a rich set of experimental data (Weiner and Wagner 1998; Jager and Fratzl 2000; Fratzl et al. 2004; Gupta et al. 2006; Fratzl and Weinkamer 2007; Fratzl 2008b; Nudelman et al. 2010; Alexander et al. 2012), in the mineralized microfibril models with varying densities $(10 \%, 20 \%$, and $40 \%)$, the mineral deposition occurs primarily in the gap region. This observation is also consistent with the experimental finding (Nudelman et al. 2010) that the mineral nucleation point is close to the C-terminus (in the first section of the gap region, immediately after the gap/overlap transition). In their work, the authors suggest that the reason is the high concentration of positive charge, which attracts negatively charged peptides, in turn responsible for the onset of mineralization. Our model suggests that a concurrent mechanism is related to the larger voids found in this region of the fibrillar structure, which facilitates the onset of mineralization. We find that the $40 \%$ mineral density case shows more mineral deposition in the gap region compared to the experimental data (Nudelman et al. 2010). This could be due to the fact that in the experimental work the mineral density profile is measured for collagen that is mineralized for $24 \mathrm{~h}$. Our model shows that the size of the mineral platelets in the gap region is $\approx 15 \times 3 \times 1.6 \mathrm{~nm}^{3}$ (for $40 \%$ mineral density). This is in good agreement with the experimental work that has shown that the size of mineral platelets is 15-55 × 5-25 × 2-3 $\mathrm{nm}^{3}$ (Fratzl et al. 1991; Nudelman et al. 2010; Alexander et al. 2012). Recent experiments (Alexander et al. 2012) show that the major fraction of the mineral in bone is outside the fibrils; this is substantiated by the presence of extrafibrillar bioapatite in nonmineralized fibrils. The inclusion
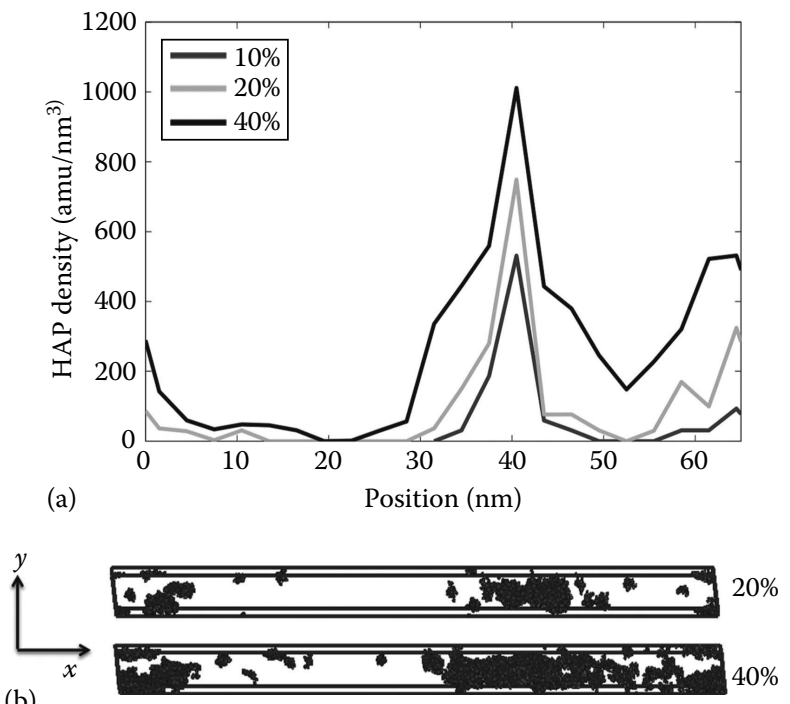

(b)

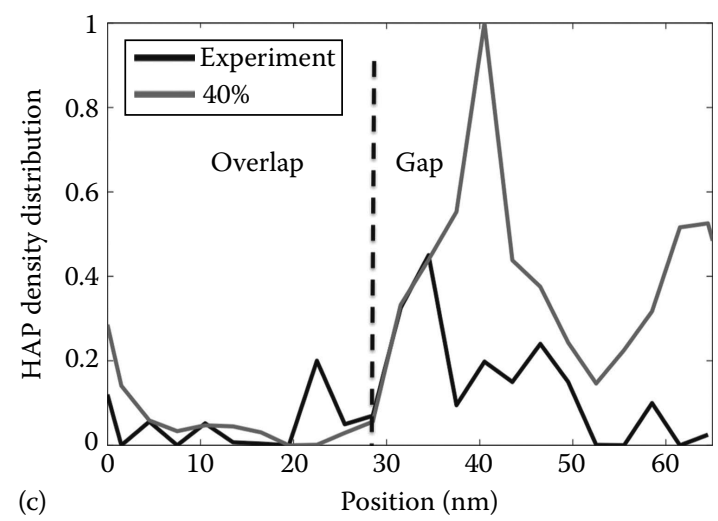

Figure 21.8 Mineral distribution in the collagen microfibril at different mineralization stages (Nair et al. 2013): (a) Distribution of HAP along the collagen fibril axis. The data shows that the maximum amount of HAP is deposited in the gap region (between 30 and $50 \mathrm{~nm}$ ). (b) Spatial distribution of HAP in the unit cell for $20 \%$ and $40 \%$ mineral densities. (c) HAP density distribution along the fibril axis for the $40 \%$ case normalized (same data as depicted in panel (a)) compared to experimental data. (From Nudelman, F. et al., Nat. Mater., 9(12), 1004, 2010.) The comparison confirms that maximum deposition is found in the gap region. (Reprinted from Nair, A.K. et al., Nat. Commun., 4, 1724, 2013.) 
of the extrafibrillar mineral content into the three-dimensional atomistic model is a challenging problem, considering the periodicity of the collagen microfibril model as well as the computational cost associated to perform the mechanical testing.

An $N V T$ ensemble is used to first equilibrate the samples $(0 \%, 20 \%$, and $40 \%)$. In order to assess the mechanical properties of mineralized collagen fibrils, we perform stress-controlled (NPT or isothermal-isobaric, a canonical ensemble where moles, $N$, pressure, $P$, and temperature, $T$, are conserved) molecular dynamics simulations with increasing tensile stress applied along the $x$-axis of the unit cell as depicted in Figure 21.9a (Nair et al. 2013). The unit cell is under constant atmospheric pressure along the other two axes $(y$ and $z$ ). We use an NPT ensemble (Plimpton 1995) for loading the samples with different stress states

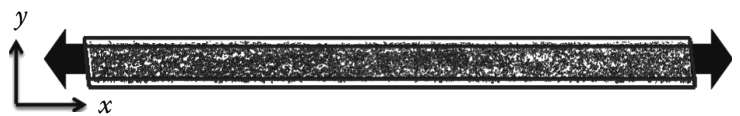

(a)
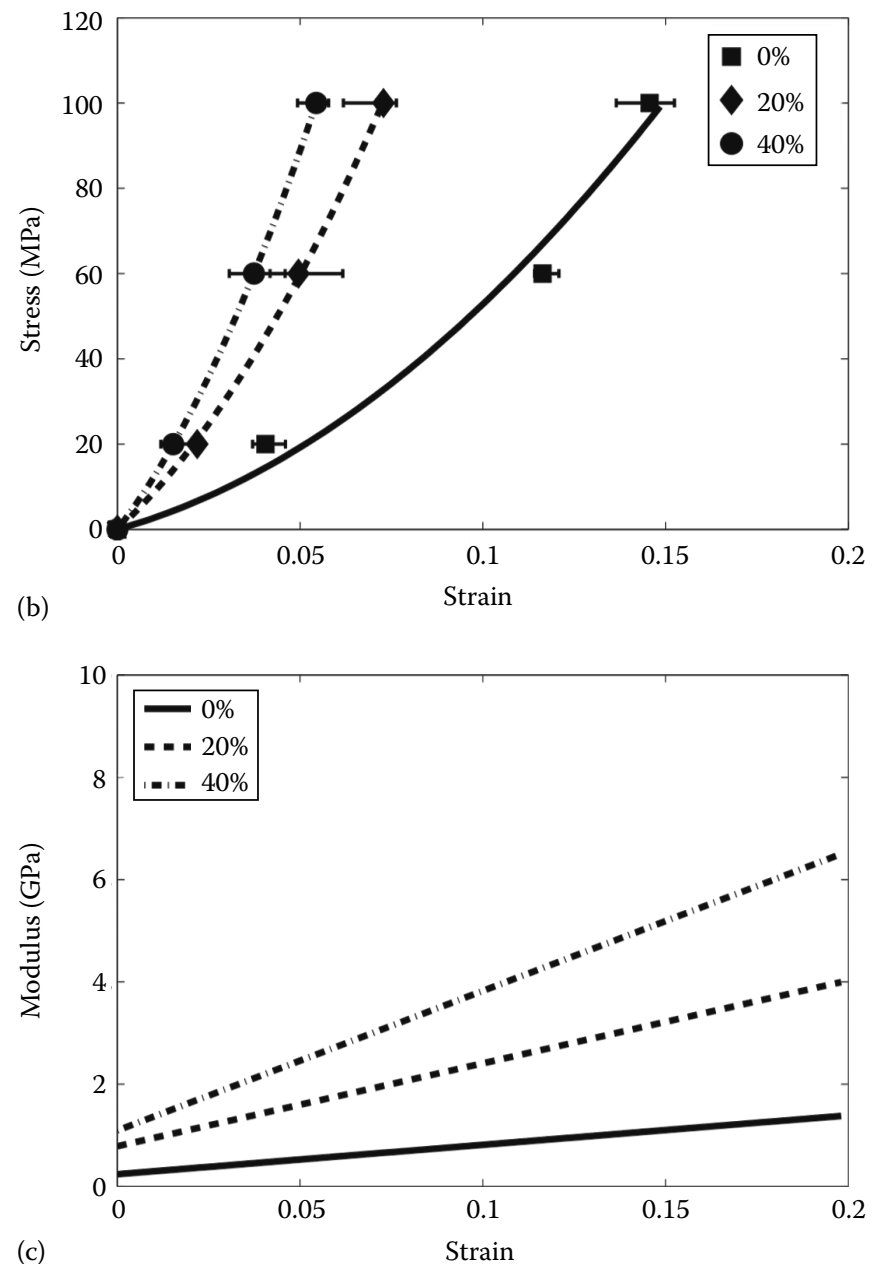

Figure 21.9 Mechanical properties of collagen fibrils at different mineralization stages as presented by Nair et al. (2013): (a) Fibril unit cell with mineral content used to perform tensile test by measuring stress versus strain. (b) Stress-strain plots for nonmineralized collagen fibril (0\%), $20 \%$ mineral density and $40 \%$ mineral density cases. (c) Modulus versus strain for $0 \%, 20 \%$, and $40 \%$ mineral densities showing an increase in modulus as the mineral content increases. (Reprinted from Nair, A.K. et al., Nat. Commun., 4, 1724, 2013.)
$(\sigma=-P)$ ranging from (1) atmospheric pressure, (2) $20 \mathrm{MPa},(3)$ $60 \mathrm{MPa}$, and (4) $100 \mathrm{MPa}$. We observe that the samples reached equilibration under applied load at approximately $6 \mathrm{~ns}$. The strain is computed as $\varepsilon=\left(L-L_{0}\right) / L_{0}$, where $L_{o}$ is the equilibrium length identified at atmospheric pressure. To ensure that equilibrium is achieved, we monitor the pressure at equilibrium, the RMSD (root mean square deviation), and confirm that the size of the simulation cell reaches a steady-state value. Using the fibril strain $\varepsilon$ associated with each applied stress $\sigma$, we obtain the stressstrain behavior for each case by plotting $\sigma$ over $\varepsilon$. The modulus is computed from the first derivative of a polynomial function that is fitted to the stress-strain data. The computational time requirement for equilibration is $0.2 \mathrm{~ns} /$ week, using 24 processors for the $40 \%$ mineral density case.

As observed from Figure 21.9b, as the mineral content increases, the stress-strain behavior of the mineralized collagen microfibril also changes compared to pure collagen fibrils, with the mineralized cases showing an increasingly higher modulus as higher mineral densities are reached. In order to quantify the variation of the modulus for different strain levels, we plot the modulus as a function of strain, as shown in Figure 21.9c. The $0 \%$ case has an initial modulus of $0.5 \mathrm{GPa}$ at a load less than $20 \mathrm{MPa}$, and increases to $1.1 \mathrm{GPa}$ as the stress increases to 100 $\mathrm{MPa}$. For larger deformation, the modulus approaches $2 \mathrm{GPa}$. These moduli are well within the range of values reported for collagen fibrils under tensile loading using both experiments and simulations (Van Der Rijt et al. 2006; Gautieri et al. 2011). For the $20 \%$ mineral density case, the initial modulus is $1.3 \mathrm{GPa}$ (stress less than $20 \mathrm{MPa}$ ), and increases to $2.7 \mathrm{GPa}$ at $100 \mathrm{MPa}$. This shows that even a relatively small mineral content greatly alters the stress-strain behavior of the collagen microfibril model and increases its modulus by $\approx 150 \%$. Similarly, the $40 \%$ mineral density case has an initial modulus of $1.5 \mathrm{GPa}$, approaching 2.8 GPa. For stresses at $100 \mathrm{MPa}$, the $20 \%$ and $40 \%$ mineral density cases have very similar tangent moduli. However, as shown in Figure $21.9 \mathrm{c}$, as the strain increases beyond $10 \%$, the modulus for the $40 \%$ mineral density case also increases, indicating that at higher strains, the mineral content provides additional stiffness to the collagen-HAP composite. The moduli identified here for the cases with $20 \%$ and $40 \%$ mineral content are consistent with a recent experimental study (Hang and Barber 2011), which showed that mineralized collagen fibrils from antler had a modulus of $2.38 \pm 0.37 \mathrm{GPa}$ for strains less than $4 \%$.

To understand the deformation mechanism for mineralized and nonmineralized samples at different deformation states, we compute the deformation fields within the fibrils. We observe that as the loading increases, there is no significant movement or coalescence of the HAP crystals in the gap region when the loading increases from 20 to $100 \mathrm{MPa}$. However, for the $0 \%$ case, the collagen molecule undergoes significant deformation in the gap region as the applied stress increases. We compute the deformation in the collagen microfibril for all the three cases at applied stresses of 20,60, and $100 \mathrm{MPa}$. We find that (Nair et al. 2013) the gap-to-overlap ratio for $0 \%$ case increases with the applied stress indicating that for pure collagen the gap region deforms significantly compared to the overlap region to accommodate the external load. This behavior is consistent with the earlier tensile tests on collagen microfibril (Gautieri 
et al. 2011). Clearly, the presence of HAP alters the deformation mechanism of the collagen fibril. For the $20 \%$ mineral density case, the gap-to-overlap ratio is nearly constant for increases in applied stress, whereas for the $40 \%$ case the gap-to-overlap ratio decreases as the applied stress increases. This shows that a higher mineral content leads to more deformation in the overlap region compared to the gap region, where the interaction between HAP and collagen limits the deformation within collagen molecules. This analysis is consistent with the results reviewed in Section 21.3.1 and also explains how energy dissipation in bone occurs at the nanoscale, leading to high toughness. We also observe that HAP takes approximately four times more stress than the collagen phase (Nair et al. 2013), while the strain in the collagen phase is two orders of magnitude higher than the HAP phase. This finding that the strain in the collagen phase is lower than the total strain is consistent with the earlier studies (Gupta et al. 2006). The three-dimensional fully atomistic microfibril model discussed in this section has great potential for future studies, in particular, related to the effect of collagen mutations on the mechanical properties of bone, for example, in diseases such as osteogenesis imperfecta (brittle bone disease).

\subsubsection{COARSE GRAIN MODELING OF BIOMINERALS}

In order to gain a more complete understanding of the mechanical behavior of bone and biomineralized materials, one must also consider its mechanical characteristics at the meso- and macroscopic length scales. This is the scale at which cracks and flaws often initiate, and where many physiological functions of mineralized biomaterials take place. It is thus clearly a critical length scale at which material performance should be investigated. Full atomistic modeling techniques are, as has been highlighted earlier in this chapter (Sections 21.3.1 through 21.3.3), very

useful and essential for understanding the nanoscale mechanisms. However, requirement of enormous computational power, especially for the model described in Section 21.3.3, restricts its applicability to the meso and macroscale phenomena. At this scale, coarse-grained techniques are very useful and potentially very insightful. This modeling technique commonly incorporates atomistic information and can have significant predictive power or at least provide mechanistic insights through comparative studies (Muller-Plathe 2002; Gautieri et al. 2010; Sen and Buehler 2011). When appropriately constructed, coarse-grained models can reach scales far beyond the atomistic scale and provide valuable insights (Muller-Plathe 2002; Gautieri et al. 2010; Sen and Buehler 2010; Dimas and Buehler 2012).

Here, we review the results of a recent study (Dimas and Buehler 2012) to illustrate how biomineralized materials can be studied effectively with coarse-grained models (Sen and Buehler 2011). Noting that biomineralized structures are commonly assembled with specific architectural arrangements, we hypothesize that these geometric arrangements are successful mechanical designs, independent of the details of the constituent materials (Dimas and Buehler 2012). Specifically, we believe that these geometric arrangements act to reduce stress concentrations around critical flaws and stabilize the propagation of fracture. Further, we hypothesize that they are capable of doing so in the absence of complex energy, dissipating proteinaceous layers and additional levels of hierarchy (Ji and Gao 2004a; Sen and Buehler 2011; Dimas and Buehler 2012).

We employ an atomistically informed spring bead model aimed to represent the common heterogeneous nature of biomineralized structures (Dimas and Buehler 2012). The spring bead model is set up with a triangular lattice, as the one shown in Figure 21.10d. Spring bead models with triangular lattices as the one presented have been validated in several independent studies of fracture in

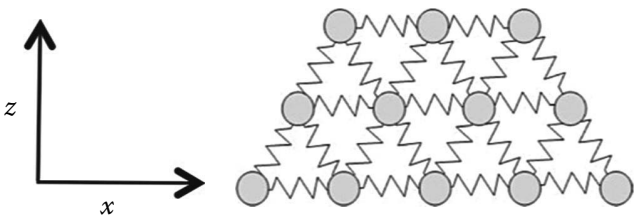
$6.48 \mu \mathrm{m}$

(a)

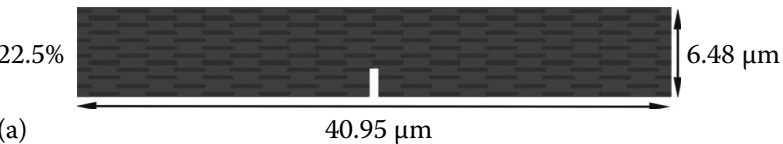

Bone-like

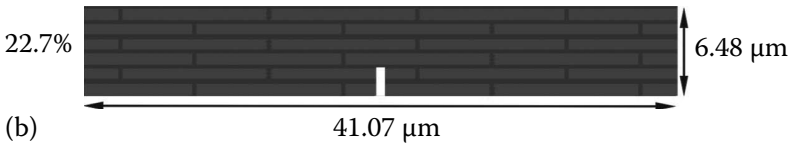

Rotated bone-like

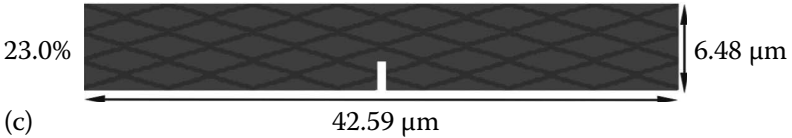

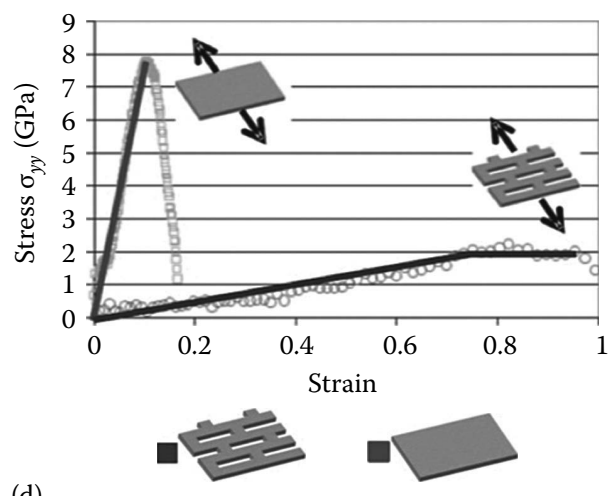

(d)

Figure 21.10 Geometries of specimens, here shown with size parameter $h=6.48 \mu \mathrm{m}$ (Dimas and Buehler 2012). With the dark and lighter phases representing the nanoporous and bulk silica, respectively, for (a) the biocalcite-like geometry, (b) the bonelike geometry, and (c) the rotated bone-like geometry. The specimens are loaded by imposing stepwise displacement on their right vertical phases, while holding the left-hand sides still. Periodic boundary conditions are employed in the horizontal direction. (d) Constitutive laws for the nanoporous and bulk silica phase representing the compliant and brittle phase, respectively in the material model. (Reprinted by permission from Macmillan Publishers Ltd. Sci. Rep., Sen, D. and Buehler, M.J. Structural hierarchies define toughness and defect-tolerance despite simple and mechanically inferior brittle building blocks, Sci. Rep., 1, 2011, Copyright 2011.) as well as the triangular lattice configuration of a spring bead model. (Reprinted from Dimas, L.S. and Buehler, M.J., Bioinspir. Biomim., 7(3), 036024, 2012.) 
heterogeneous materials at the meso- and macroscale (Hassold and Srolovitz 1989; Curtin and Scher 1990; Gao and Klein 1998). The specific force extension laws employed in this study are the ones also presented in Figure 21.10d. These behaviors represent the nanoscale deformation characteristics of bulk and nanoporous silica (Sen and Buehler 2010). The choice of silica as the base constituent of our models is motivated by the aim of making our models as general as possible. We seek to improve the mechanical characteristics of an inferior and brittle base constituent simply by tuning the geometrical features. The softer phase also consists solely of silica and achieves its compliance due to the geometry and nanoconfinement (Garcia et al. 2011).

The goal of this study is to investigate the improved mechanical characteristics achieved through ordering a soft and stiff phase in an appropriate configuration. We therefore choose to isolate this mechanism in our models, motivating the simple model described earlier. We exclude other features accounting for other mechanically significant mechanisms (e.g., microcracking, crack-bridging, etc.), which have been comprehensively studied by others (Weiner and Wagner 1998; Aizenberg et al. 2005; Weaver et al. 2007; Meyers et al. 2008), in order to isolate the effect of architecture and recognize the limitations; this enforces on the predictive power of our models.

Inspired by natural materials, we investigate two specific arrangements of the softer and stiffer phase, a biocalcite-like geometry and a bone-like geometry, reviewing here what was reported in a recent study (Dimas and Buehler 2012). Further, in an attempt to optimize the strain transfer, we construct and investigate an additional geometry, here named rotated-bone-like geometry. All geometries are presented with appropriate titles in Figure 21.10. In order to study the mechanics of these structures, it is essential to evaluate the local variation of stresses and strains. Although these concepts are somewhat less familiar in the context of discrete particles, well-defined expressions do exist that have been shown to be equivalent to the continuum measures, under the assumption of validity of the Cauchy-Born rule. To evaluate stresses, we employ the virial stress measure presented in the study of Tsai (1979). For characterization of the local variation of strains, we find the measure developed by Zimmerman et al. (2009) useful. The strain is defined through the definition of the left Cauchy-Green tensor, unique to a particular particle in terms of its nearest neighbors:

$$
b_{i j}^{l}=\frac{1}{\lambda} \sum_{k=1}^{N}\left(\frac{\Delta x_{i}^{k l} \Delta x_{j}^{k l}}{r_{0}^{2}}\right) .
$$

Here, $\Delta x_{i}^{k l}=x_{i}^{l}-x_{i}^{k}$ and $\Delta x_{j}^{k l}=x_{j}^{l}-x_{j}^{k}$, with $x_{i}^{l}$ representing the $i$ th component of the coordinates of atom $l$ in the deformed configuration. Further, $r_{0}$ is the equilibrium spacing of the lattice, $N$ is the total number of nearest neighbors, and $\lambda$ is a prefactor depending on the specific lattice chosen. For this case with the triangular lattice with nearest neighbor interactions, $\lambda=3$. We recall that the left Cauchy-Green tensor is a symmetric positive definite matrix and hence, as a spectral decomposition, an engineering strain is calculated, and the specific form employed is

$$
\underline{\underline{\varepsilon}}=\sqrt{\underline{\underline{b}}}-\underline{\underline{1}} .
$$

Here, 1 is the identity tensor, and the square root of a symmetric positive definite matrix is calculated by

$$
\sqrt{\underline{\underline{b}}}=\sum_{i}^{3} \sqrt{w_{i}} \cdot \underline{e_{i}} \otimes \underline{e_{i}}
$$

where

$$
\underline{\underline{b}}=\sum_{i}^{3} w_{i} \cdot \underline{e_{i}} \otimes \underline{e_{i}}
$$

and $w_{i}$ and $\underline{e_{i}}$ are the eigenvalues and eigenvectors of the left Cauchy-Green tensor, respectively. This strain is a purely geometric measure and strictly only be applicable away from surfaces and interfaces. In our study, we use this strain measure to make qualitative statements and comparisons and, thus, deem this measure suitable. The computed stresses and strains are evaluated by visualization through stress and strain fields plotted in MATLAB.

In Figure 21.11, we present stress field plots of unnotched and notched bulk silica and unnotched and notched bone-likecomposites of bulk nanoporous silica. Figure 21.12 displays longitudinal and shear strain plots of unnotched and notched samples of the same bone-like composites. Figure 21.11a and c clearly shows the brittle nature of silica and the very high sensitivity to cracks that it exhibits. The nature of the stress field is completely changed by the introduction of the crack, and it is clear to see that the effective strength of the specimen is greatly reduced by the introduction of the flaw. These images are strongly contrasted by their neighbors in Figure $21.11 \mathrm{~b}$ and d. The architecture of the composite introduces a distinct pattern in the stress distribution that is interestingly enough upheld, despite the introduction of the crack in Figure 21.11d, albeit at somewhat lower levels of stress. The soft nature of the matrix phase forces deformations and stresses to delocalize from the tip of the flaw (see also Figure 21.12). Stiff platelets far from the flawed region maintain significant levels of stress due to the geometrical configuration of the structure. Indeed, the geometry also forces the small vertical regions of the matrix phase to participate in stress transfer, thereby giving an overall efficient material.

\subsection{OUTLOOK}

In this chapter, we presented atomistic models applied to study the interactions between collagen and the biomineral HAP at the nanoscale, in various configurations, and from a molecular point of view that connected the chemical composition to mechanical properties (Dimas and Buehler 2012; Qin et al. 2012; Libonati et al. 2013; Nair et al. 2013). The coarse grain model discussed originally in the work of Dimas and Buehler (2012) allowed us to examine the fracture properties of biominerals at the macroscale and provide an integrated view of mechanisms occurring at multiple scales. The models may help us to better understand the in vivo structural and mechanical behavior of bone, and can provide solutions to various diseases with respect to mineral content and mutation in collagen. With the 


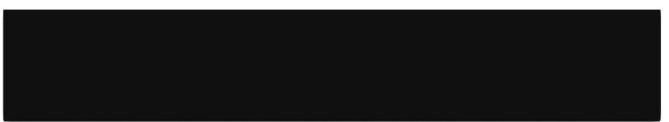

(a)

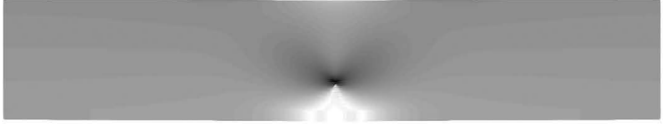

(c)

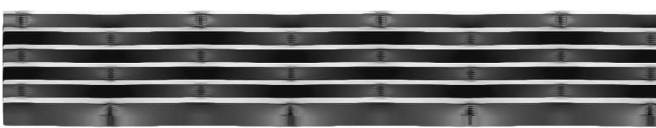

(b)

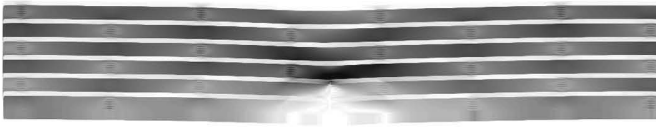

(d)
$5000 \mathrm{MPa}$

$4000 \mathrm{MPa}$

$3000 \mathrm{MPa}$

$2000 \mathrm{MPa}$

$1000 \mathrm{MPa}$

$0 \mathrm{MPa}$

Figure 21.11 Von Mises stress fields for $6.48 \mu \mathrm{m}$ (a) unnotched bulk silica, (b) unnotched bone-like, (c) notched bulk silica, and (d) notched bone-like specimens at the instant immediately prior to failure (Dimas and Buehler 2012). The unnotched bulk silica specimen shows the expected even distribution of stress throughout the sample, while the notched specimen exhibits the strong characteristic stress concentration at the crack tip. The unnotched bone-like specimen exhibits a clearly larger stress state than the notched specimen. However, the load path in both specimens is seen to be very similar. This specific hierarchical geometry alleviates the stress tip concentration and maintains the same mechanism of load transfer, despite the presence of the crack, thus reducing the specimen's sensitivity to the notch. (Reprinted from Dimas, L.S. and Buehler, M.J., Bioinspir. Biomim., 7(3), 036024, 2012.)

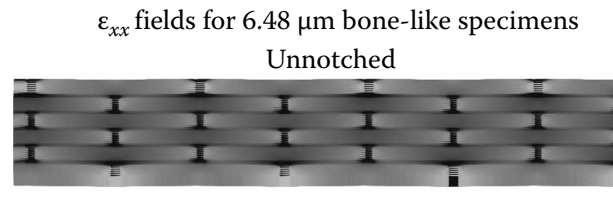

(a)

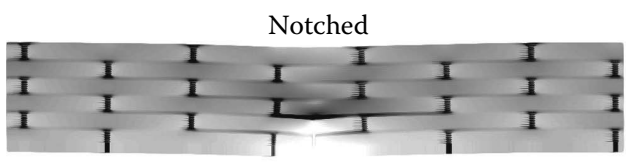

(c)

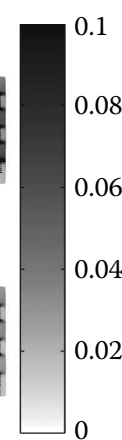

$\varepsilon_{x z}$ fields for $6.48 \mu \mathrm{m}$ bone-like specimens

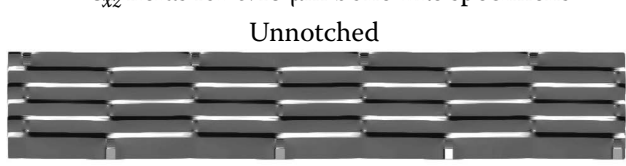

(b)

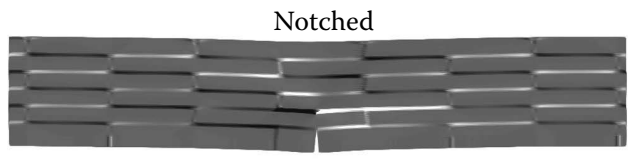

(d)

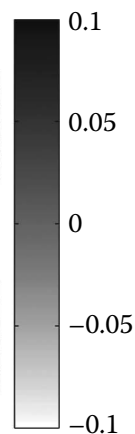

$-0.1$

Figure 21.12 (a) Longitudinal and (b) shear strain fields for unnotched bone-like geometry at the instant immediately prior to failure (Dimas and Buehler 2012). (c) Longitudinal and (d) shear strain fields for notched bone-like geometry at the instant immediately prior to failure. Whereas the longitudinal strain transfer is significantly impeded in the stiff phase by the introduction of the crack, the panels clearly show that the strain field in the soft compliant phase remains virtually unchanged. (Reprinted from Dimas, L.S. and Buehler, M.J., Bioinspir. Biomim., 7(3), 036024, 2012.)

availability of additive manufacturing, the coarse-grained model predictions can also be useful to create new materials that mimic the structural hierarchies of biominerals, as discussed in a recent study (Dimas et al. 2013). The predictions from these studies can also be useful to provide design criteria for the development of biomineral-based composites with enhanced mechanical properties.

\section{REFERENCES}

Aizenberg, J., J.C. Weaver et al. (2005). Skeleton of Euplectella sp.: Structural hierarchy from the nanoscale to the macroscale. Science 309(5732): 275-278.

Alexander, B., T.L. Daulton et al. (2012). The nanometre-scale physiology of bone: Steric modelling and scanning transmission electron microscopy of collagen-mineral structure. J Roy Soc Interf 9 (73): 1774-1786.

Allegrini, S., Jr., E. Rumpel et al. (2006). Hydroxyapatite grafting promotes new bone formation and osseointegration of smooth titanium implants. Ann Anat 188(2): 143-151.

Almora-Barrios, N. and N.H. de Leeuw (2010a). A density functional theory study of the interaction of collagen peptides with hydroxyapatite surfaces. Langmuir 26(18): 14535-14542.
Almora-Barrios, N. and N.H. de Leeuw (2010b). Modelling the interaction of a Hyp-Pro-Gly peptide with hydroxyapatite surfaces in aqueous environment. Cryst Eng Comm 12(3): 960-967.

Bhowmik, R., K.S. Katti et al. (2007a). Molecular dynamics simulation of hydroxyapatite-polyacrylic acid interfaces. Polymer 48(2): 664-674.

Bhowmik, R., K.S. Katti et al. (2007b). Mechanics of molecular collagen is influenced by hydroxyapatite in natural bone. J Mater Sci $\mathbf{4 2 ( 2 1 )}$ : 8795-8803.

Bhowmik, R., K.S. Katti et al. (2009). Mechanisms of load-deformation behavior of molecular collagen in hydroxyapatite-tropocollagen molecular system: Steered molecular dynamics study. J Eng MechASCE 135(5): 413-421.

Boskey, A. (2003). Bone mineral crystal size. Osteop Int 14: S16-S20.

Brooks, B.R., R.E. Bruccoleri et al. (1983). Charmm-A program for macromolecular energy, minimization, and dynamics calculations. J Comp Chem 4(2): 187-217.

Buehler, M.J. (2006). Atomistic and continuum modeling of mechanical properties of collagen: Elasticity, fracture, and self-assembly. J Mater Res 21(8): 1947-1961.

Buehler, M.J. (2007). Molecular nanomechanics of nascent bone: Fibrillar toughening by mineralization. Nanotechnology 18: 295102.

Buehler, M.J., H. Yao et al. (2006). Cracking and adhesion at small scales: Atomistic and continuum studies of flaw tolerant nanostructures. Model Simul Mater Sci Eng 14(5): 799. 
Colfen, H. (2010). Biomineralization: A crystal-clear view. Nat Mater 9(12): 960-961.

Curtin, W.A. and H. Scher (1990). Mechanics modeling using a spring network. J Mater Res 5(3): 554-562.

Dimas, L., G. Bratzel et al. (2013). Tough composites inspired by mineralized natural materials: Computation, $3 \mathrm{D}$ printing and testing. Adv Funct Mater 23(36): 4629-4638.

Dimas, L.S. and M.J. Buehler (2012). Influence of geometry on mechanical properties of bio-inspired silica-based hierarchical materials. Bioinspir Biomim 7(3): 036024.

Dubey, D.K. and V. Tomar (2008). Microstructure dependent dynamic fracture analyses of trabecular bone based on nascent bone atomistic simulations. Mech Res Commun 35(1-2): 24-31.

Fratzl, P. (2008a). Bone fracture-When the cracks begin to show. Nat Mater 7(8): 610-612.

Fratzl, P. ed. (2008b). Collagen: Structure and Mechanics, Springer, New York.

Fratzl, P., N. Fratzl-Zelman et al. (1991). Nucleation and growth of mineral crystals in bone studied by small-angle X-ray scattering. Calc Tiss Int 48(6): 407-413.

Fratzl, P., H.S. Gupta et al. (2004). Structure and mechanical quality of the collagen-mineral nano-composite in bone. J Mater Chem 14(14): 2115-2123.

Fratzl, P. and R. Weinkamer (2007). Nature's hierarchical materials. Progr Mater Sci 52(8): 1263-1334.

Gao, H. (2006). Application of fracture mechanics concepts to hierarchical biomechanics of bone and bone-like materials. In Advances in Fracture Research, eds. A. Carpinteri, Y.-W. Mai, and R. Ritchie, Springer, The Netherlands, pp. 101-137.

Gao, H., B. Ji et al. (2003). Materials become insensitive to flaws at nanoscale: Lessons from nature. Proc Natl Acad Sci 100(10): 5597-5600.

Gao, H.J. and P. Klein (1998). Numerical simulation of crack growth in an isotropic solid with randomized internal cohesive bonds. J Mech Phys Solids 46(2): 187-218.

Garcia, A.P., D. Sen et al. (2011). Hierarchical silica nanostructures inspired by diatom algae yield superior deformability, toughness, and strength. Metallurg Mater Trans A-Phys Metallurg Mater Sci 42A(13): 3889-3897.

Gautieri, A., M.J. Buehler et al. (2009a). Deformation rate controls elasticity and unfolding pathway of single tropocollagen molecules. J Mech Behav Biomed Mater 2(2): 130-137.

Gautieri, A., A. Russo et al. (2010). Coarse-grained model of collagen molecules using an extended MARTINI force field. J Chem Theory Computat 6(4): 1210-1218.

Gautieri, A., S. Uzel et al. (2009b). Molecular and mesoscale mechanisms of osteogenesis imperfecta disease in collagen fibrils. Biophys $J$ 97(3): 857-865.

Gautieri, A., S. Vesentini et al. (2009c). Intermolecular slip mechanism in tropocollagen nanofibrils. Int J Mater Res 100(7): 921-925.

Gautieri, A., S. Vesentini et al. (2011). Hierarchical structure and nanomechanics of collagen microfibrils from the atomistic scale up. Nano Lett 11(2): 757-766.

Gupta, H.S., S. Krauss et al. (2013). Intrafibrillar plasticity through mineral/collagen sliding is the dominant mechanism for the extreme toughness of antler bone. J Mech Behav Biomed Mater. http://dx.doi.org/10.1016/j.jmbbm.2013.03.020.

Gupta, H.S., J. Seto et al. (2006). Cooperative deformation of mineral and collagen in bone at the nanoscale. Proc Natl Acad Sci USA 103(47): 17741-17746.

Gupta, H.S., W. Wagermaier et al. (2005). Nanoscale deformation mechanisms in bone. Nano Lett 5(10): 2108-2111.

Hang, F. and A.H. Barber (2011). Nano-mechanical properties of individual mineralized collagen fibrils from bone tissue. J Roy Soc Interf 8(57): 500-505.

Hassold, G.N. and D.J. Srolovitz (1989). Brittle-fracture in materials with random defects. Phys Rev B 39(13): 9273-9281.
Hauptmann, S., H. Dufner et al. (2003). Potential energy function for apatites. Phys Chem Chem Phys 5(3): 635-639.

Hu, Y.Y., A. Rawal et al. (2010). Strongly bound citrate stabilizes the apatite nanocrystals in bone. Proc Natl Acad Sci USA 107(52): 22425-22429.

Humphrey, W., A. Dalke et al. (1996). VMD: Visual molecular dynamics. J Mol Graph 14(1): 27-38.

Jager, I. and P. Fratzl (2000). Mineralized collagen fibrils: A mechanical model with a staggered arrangement of mineral particles. Biophys $J$ 79(4): 1737-1746.

Ji, B.H. and H.J. Gao (2004a). Mechanical properties of nanostructure of biological materials. J Mech Phys Solids 52(9): 1963-1990.

Ji, B.H. and H.J. Gao (2004b). A study of fracture mechanisms in biological nano-composites via the virtual internal bond model. Mater Sci Eng A-Struct Mater Prop Microstruct Process 366(1): 96-103.

Katti, D.R., S.M. Pradhan et al. (2010). Directional dependence of hydroxyapatite-collagen interactions on mechanics of collagen. J Biomech 43(9): 1723-1730.

Kundu, B., A. Lemos et al. (2010). Development of porous HAp and beta-TCP scaffolds by starch consolidation with foaming method and drug-chitosan bilayered scaffold based drug delivery system. J Mater Sci Mater Med 21(11): 2955-2969.

Launey, M.E., M.J. Buehler et al. (2010). On the mechanistic origins of toughness in bone. In Annual Review of Materials Research, Vol. 40. eds. D.R. Clarke, M. Ruhle, and F. Zok, Palo Alto, CA: Annual Reviews, pp. 25-53.

Li, J. (2003). AtomEye: An efficient atomistic configuration viewer. Model Simul Mater Sci Eng 11(2): 173.

Libonati, F., A.K. Nair et al. (2013). Fracture mechanics of hydroxyapatite single crystals under geometric confinement. J Mech Behav Biomed Mater 20: 184-191.

Meyers, M.A., P.Y. Chen et al. (2008). Biological materials: Structure and mechanical properties. Progr Mater Sci 53(1): 1-206.

Muller-Plathe, F. (2002). Coarse-graining in polymer simulation: From the atomistic to the mesoscopic scale and back. Chemphyschem 3(9): 754-769.

Nair, A.K., A. Gautieri et al. (2013). Molecular mechanics of mineralized collagen fibrils in bone. Nat Commun 4: 1724.

Nudelman, F., K. Pieterse et al. (2010). The role of collagen in bone apatite formation in the presence of hydroxyapatite nucleation inhibitors. Nat Mater 9(12): 1004-1009.

Park, S., R.J. Radmer et al. (2005). A new set of molecular mechanics parameters for hydroxyproline and its use in molecular dynamics simulations of collagen-like peptides. J Comput Chem 26(15): $1612-1616$.

Plimpton, S. (1995). Fast parallel algorithms for short-range moleculardynamics. J Computat Phys 117(1): 1-19.

Qin, Z., A. Gautieri et al. (2012). Thickness of hydroxyapatite nanocrystal controls mechanical properties of the collagen-hydroxyapatite interface. Langmuir 28(4): 1982-1992.

Qin, Z., L. Kreplak et al. (2009). Hierarchical structure controls nanomechanical properties of vimentin intermediate filaments. PLoS ONE 4(10): e7294.

Rainey, J.K. and M.C. Goh (2004). An interactive triple-helical collagen builder. Bioinformatics 20(15): 2458-2459.

Rho, J.-Y., L. Kuhn-Spearing et al. (1998). Mechanical properties and the hierarchical structure of bone. Med Eng Phys 20(2): 92-102.

Ritchie, R.O., M.J. Buehler et al. (2009). Plasticity and toughness in bone. Phys Today 62(6): 41-47.

Ritchie, R.O., J.J. Kruzic et al. (2004). Characteristic dimensions and the micro-mechanisms of fracture and fatigue in 'nano' and 'bio' materials. Int J Fract 128(1): 1-15.

Sen, D. and M.J. Buehler (2010). Atomistically-informed mesoscale model of deformation and failure of bioinspired hierarchical silica nanocomposites. Int J Appl Mech 2(4): 699-717. 
Sen, D. and M.J. Buehler (2011). Structural hierarchies define toughness and defect-tolerance despite simple and mechanically inferior brittle building blocks. Sci Reports 1: Article No. 35.

Snyders, R., D. Music et al. (2007). Experimental and ab initio study of the mechanical properties of hydroxyapatite. Appl Phys Lett 90(19).

Sopyan, I., M. Mel et al. (2007). Porous hydroxyapatite for artificial bone applications. Sci Technol Adv Mater 8(1-2): 116-123.

Tsai, D.H. (1979). Virial theorem and stress calculation in moleculardynamics. J Chem Phys 70(3): 1375-1382.

Van Der Rijt, J.A.J., K.O. Van Der Werf et al. (2006). Micromechanical testing of individual collagen fibrils. Macromol Biosci 6(9): 699-702.

Weaver, J.C., J. Aizenberg et al. (2007). Hierarchical assembly of the siliceous skeletal lattice of the hexactinellid sponge Euplectella aspergillum. J Struct Biol 158(1): 93-106.
Weiner, S. and H.D. Wagner (1998). The material bone: Structure mechanical function relations. Ann Rev Mater Sci 28: 271-298.

Zahn, D. and P. Duchstein (2011). Atomistic modeling of apatitecollagen composites from molecular dynamics simulations extended to hyperspace. J Mol Model 17(1): 73-79.

Zhao Q., A. Gautieri et al. (2012). Thickness of hydroxyapatite nanocrystal controls mechanical properties of the collagenhydroxyapatite interface. Langmuir 28: 1982-1992.

Zimmerman, J.A., D.J. Bammann et al. (2009). Deformation gradients for continuum mechanical analysis of atomistic simulations. Int $J$ Solids Struct 46(2): 238-253.

Zimmerman, J.A., E.B. Webb III et al. (2004). Calculation of stress in atomistic simulation. Model Simul Mater Sci Eng 12(4): S319. 